\title{
Ausschreibung des Preises „Edgard Milhaud“ 2010
}

Seit 1902 Inhaber des Lehrstuhls für politische Ökonomie an der Universität Genf und Gründer der Zeitschrift „Die Annalen der Gemeinwirtschaft" trat Edgard Milhaud durch seine Schriften und seine Tätigkeit beim Internationalen Arbeitsamt (IAA) für eine bessere soziale und wirtschaftliche Organisation in der Welt mit dem Ziel der Sicherung des Weltfriedens ein.

Mit 75 Jahren setzte sich Edgard Milhaud für einen nachhaltigen Fortbestand der wissenschaftlichen Zeitschrift ein. Dank der aktiven Unterstützung von Schweizer Freunden gründete 1947 Milhaud das Forschungs- und Informationszentrum CIRIEC, um Untersuchungen in verschiedenen Ländern durchzuführen. Ihm schwebte sogar vor, dass daraus eine internationale Nichtregierungsorganisation entstehen könnte, in der sozioökonomische Themen offen debattiert werden könnten nach dem Vorbild der Internationalen Vereinigung für Sozialen Fortschrift, die am Rande des IAA gegründet wurde.

\section{Artikel 1 - Der Preis „Edgard Milhaud“6}

In Erinnerung an seinen Gründer richtet das Internationale Forschungs- und Informationszentrum für öffentliche Wirtschaft, Sozialwirtschaft und Genossenschaftswesen (CIRIEC) den zweijährlichen internationalen Preis „Edgard Milhaud“ gemäß der Beschlussfassung seines Internationalen Vorstands vom 5. Dezember 2008 ein.

Der Preis „Edgard Milhaud“ ist als Anreiz und als Auszeichnung für die Arbeit eines Nachwuchsforschers oder eines jungen Experten zu einem für das CIRIEC relevanten Thema mit inhaltlichem Bezug auf die zweijährlichen Kongresse gedacht. Der Preis sollte für junge Leute eine Anregung sein, sich aktiv bei CIRIEC einzubringen und/oder Interesse für die Sektoren und Aktivitäten, die von CIRIEC erforscht werden, zu entwickeln. Der Arbeitsschwerpunkt von CIRIEC sind Wirtschaftsbereiche und Tätigkeiten, die auf das allgemeine und kollektive Interesse ausgerichtet sind:

- das Handeln des Staates und der regionalen und lokalen öffentlichen Hand in wirtschaftlichen Bereichen (Wirtschaftspolitik, Regulierung);

- die öffentlichen Dienstleistungen und Leistungen der Daseinsvorsorge sowie die öffentlichen und gemischtwirtschaftlichen Unternehmen auf nationaler, regionaler und lokaler Ebene;

- die Sozialwirtschaft: Genossenschaften, Gesellschaften auf Gegenseitigkeit und gemeinnützige Vereine etc.

Für die Verleihung 2010 lautet das Thema „Öffentliche und soziale Wirtschaft in der Wirtschaftskrise: Ein Stabilitätsfaktor in ökonomischen Krisen und Stütze einer nachhaltigen Entwicklung der Volkswirtschaften“. 


\section{Artikel 2 - Die Jury}

Den Vorsitz der Preisvergabe-Jury führt Frau Leona DETIEGE, Ehrenpräsidentin des CIRIEC.

Neben der Präsidentin setzt sich die Jury aus den folgenden Mitgliedern zusammen:

- Benoît LÉVESQUE, Präsident des Internationalen Wissenschaftlichen Beirats, Université du Québec à Montréal, Canada

- Rafael CHAVES, Präsident der Internationalen Wissenschaftlichen Kommission „Sozialwirtschaft und Genossenschaftswesen“, Universidad de Valencia, CIRIECEspaña

- Gabriel OBERMANN, Präsident der Internationalen Wissenschaftlichen Kommission „Öffentliche Dienstleistungen/Öffentliche Unternehmen“, WU Wirtschaftsuniversität Wien, Österreich

- Fabienne FECHER, Redaktorin der Annals of Public and Cooperative Economics, Université de Liège, Belgique

- Giuseppe BOGNETTI, Università Statale di Milano, CIRIEC-Italia

- Marie BOUCHARD, Université du Québec à Montreal, Canada

- Danièle DEMOUSTIER, Institut d'études politiques de Grenoble, France

- Hajime IMAMURA, Toyo University, CIRIEC-Japan

- José Luis MONZON, Universidad de Valencia, Präsident von CIRIEC-España

- $\quad$ Frank SCHULZ-NIESWANDT, Universität zu Köln, CIRIEC-Deutschland

- Mauricio SERVA, Florianópolis, Präsident von CIRIEC-Brasil.

\section{Artikel 3 - Nominierung und Auswahl des Preisträgers}

Für die Nominierung der Kandidaten und die Auswahl des Preisträgers gelten folgende Regeln:

\section{1 - Kriterien für die Auswahl der Kandidaten}

Der Preis richtet sich an Nachwuchsforscher (unter 30 Jahre zum Stichtag 1. Januar 2010), die erst vor kurzem eine Dissertation oder eine entsprechende 3. Zyklus Arbeit mit Bezug auf das vorgeschlagene Thema verfasst haben. Außerdem müssen die Kandidaten ihr Interesse an den Tätigkeiten des CIRIEC bekunden, indem sie darlegen, welchen Beitrag sie zur Bereicherung der Arbeiten des CIRIEC leisten könnten.

Die Auswahlkriterien sind:

- wissenschaftliche Qualität

- Relevanz für und Auswirkungen auf die Gesellschaft.

Die Kandidaten werden von den nationalen Sektionen und/oder Mitgliedern des CIRIEC vorgeschlagen. 
Eine Vorauswahl wird von jeder nationalen Sektion des CIRIEC für ihre nationalen Kandidaten durchgeführt. Jede Sektion oder jedes Mitglied des CIRIEC kann höchstens zwei Kandidaten pro Land vorschlagen.

\section{2 - Praktische Aspekte}

Das Sekretariat des CIRIEC wird das Nominierungsverfahren mittels Ankündigung auf seiner Internetseite und durch Bekanntmachung in seinem Netz ein Jahr vor dem Kongress eröffnen.

Die Vorschläge für die Nominierungen müssen spätestens bis zum 30. Januar 2010 an das internationale Sekretariat des CIRIEC (Postanschrift: CIRIEC, Université de Liège au Sart-Tilman, Bât. B 33, bte 6, BE - 4000 Liège, Belgien - Email: ciriec@ulg.ac.be) gesandt werden, wo auch alle zusätzlichen Informationen eingeholt werden können.

Die Vorschläge müssen ein Begründungsschreiben und einen Lebenslauf (CV) des Kandidaten enthalten. Im CV sind die jüngsten Arbeiten des Kandidaten hervorzuheben. Ferner muss eine Kopie der Arbeit des Kandidaten für eine Bewertung beiliegen. Eine $\mathrm{Zu}-$ sammenfassung (zehn Seiten) muss auf Englisch eingereicht werden.

\section{Artikel 4 - Der Preis}

Der Preis wird alle zwei Jahre anlässlich des zweijährlichen Kongresses von CIRIEC vergeben. Der Preisträger erhält einen Preis, der 2010 mit 10.000 Euro dotiert ist.

\section{Artikel 5 - Preisverleihung}

Die Preisverleihung 2010 findet im Rahmen der Schlussveranstaltung des Internationalen Kongresses von CIRIEC am 18. Mai 2010 in Berlin statt.

Der Preis „Edgard Milhaud“ wird dem Preisträger durch den Präsidenten von CIRIEC in Anwesenheit eventueller Sponsoren verliehen. 